\title{
Comments on the paper entitled "Is post-mortem CT of the dentition adequate for correct forensic identification?: comparison of dental computed tomograpy and visual dental record" by S. Kirchhoff et al.
}

\author{
Christian Jackowski • Anders Persson
}

Received: 26 October 2009 / Accepted: 12 January 2010 / Published online: 10 February 2010

(C) Springer-Verlag 2010

\section{Dear Editor}

We would like to comment on the paper: "Is post-mortem CT of the dentition adequate for correct forensic identification?: comparison of dental computed tomograpy and visual dental record" by S. Kirchhoff et al. [1].

First of all, we want to express our gratitude to the authors for the attempt to validate the recently published technical developments in postmortem CT imaging of the dentition with a practical study.

However, the authors draw a conclusion based on their study that cannot remain uncommented. They suggest not to implement postmortem $\mathrm{CT}$ in the case of a mass casualty as it does not reach a $100 \%$ precision and the reliability of the results are affected thereby. This rather strong conclusion does not hold after a more detailed look on the methods applied in their study. The authors used a Somatom Definition CT by Siemens Medical Solutions that provides an isotropic resolution of $0.24 \mathrm{~mm}$ at its ultrahigh-resolution settings (z-Sharp ${ }^{\mathrm{TM}}$ technology). Instead of using the highest possible spatial resolution when investigating tiny and detailed specimen such as dental restorations and fillings, the authors worked with a slice thickness of $0.6 \mathrm{~mm}$. That is neither comprehensible nor reasonable. They further claim to have used the extended CT scale

\section{Jackowski $(\bowtie)$}

Institute of Legal Medicine, University of Zürich,

Winterthurerstrasse 190/52,

8057 Zürich, Switzerland

e-mail: christian.jackowski@irm.uzh.ch

\section{A. Persson}

CMIV, Center for Medical Image Science

and Visualization, University of Linköping,

Linköping,

58185 Linköping, Sweden during raw data acquisition. However, the extended CT scale is a specific way of transforming the raw data into images at the time of back projection and does not affect the raw data in any way.

Looking at the figures provided more questions arise. Why did the authors just cut parts of the streaks away (Fig. 3a) instead of deleting the Hounsfield unit range they occupy in the volume rendering presets? This possibility of improving the visualization of amalgam and metallic restorations is broadly described in the paper of Jackowski et al. (2008), which the authors refer to in their reference entry no. 15 [2].

We wish to emphasize that the methods applied in the study of Kirchhoff et al. do not represent today's state of the art of dental imaging by CT and are not a solid base for their strong conclusion.

We are convinced that in mass identification there is still a great need for objective and observer independent documentation of the corpse including the dentition and CT is certainly a feasible possibility. Although there is of course still space for further improvements of the technology, there is no reasonable argument against starting data acquisition already today.

\section{References}

1. Kirchhoff S, Fischer F, Lindemaier G et al (2008) Is post-mortem CT of the dentition adequate for correct forensic identification?: comparison of dental computed tomograpy and visual dental record. Int J Legal Med 122:471-479

2. Jackowski C, Wyss M, Persson A et al (2008) Ultra-highresolution dual-source CT for forensic dental visualizationdiscrimination of ceramic and composite fillings. Int J Legal Med 122:301-307 